\title{
De las comunidades de aprendizaje hacia una comunidad de docentes lectores.
}

From the learning communities towards a community of reading teachers.

Kelly Cristina Balmaceda Sulayvar.

Universidad de Córdoba - Colombia

balmacedakelly@gmail.com 


\section{Resumen}

La lectura crítica es una necesidad en la actualidad, por lo que se requiere poseer habilidades que permitan al individuo leer y comprender el mensaje del texto para relacionar su significado con la realidad que lo circunda. Por esta razón, se ha convertido en un reto para la escuela la formación de lectores críticos, lo cual supone que el profesorado brinde al estudiante las estrategias y herramientas que le permitan comprender diferentes tipos de texto. Partiendo de esta necesidad, y teniendo como marco el Programa Todos a Aprender (PTA) del Ministerio de Educación Nacional, se realizó un estudio desde la Investigación Acción del paradigma cualitativo que buscó transformar la comunidad de aprendizaje (CA) en una comunidad de docentes lectores. Para lograr este fin, se parte de la exploración de las necesidades frente a la lectura, luego, se aplican grupos de discusión con docentes, escenario que permitió develar las concepciones que poseían los docentes sobre la lectura, y en este mismo escenario, se realizó la socialización e implementación de varias estrategias de lectura desde una visión sociocultural, que luego fueron observadas en el desarrollo de las clases de los docentes.

\section{Abstract}

Critical reading is a necessity today, because we are in the information age and it is necessary to possess skills that allow the individual to read and understand the message of the text to relate its meaning to the reality that surrounds it. For this reason, it has become a challenge for the school to train critical readers, which means that teachers provide students with strategies and tools that allow them to understand different types of text. Based on this need, and based on the PTA of the Ministry of National Education, a study was carried out from the action research of the qualitative paradigm that sought to transform the learning community (CA) into a community of teaching readers. In order to achieve this goal, we started with an exploration of the needs of reading, then discussion groups were applied with teachers, a scenario that allowed us to unveil the conceptions that teachers had about reading, and in this same scenario, The socialization and implementation of several reading strategies from a sociocultural perspective, which were then observed in the development of the teachers' classes.

\section{Palabras Clave}

Comunidades de Aprendizaje, Lectura, Docencia, Investigación Acción.

\section{Keywords}

Communities of Learning, Reading, Teaching, Action Research. 


\section{Introducción}

En la actualidad, se enfatiza esta tarea en la formación de lectores que sean capaces de crear nuevas experiencias en torno a las prácticas de lectura. Para ello, se hace necesario generar un clima de constante reflexión y análisis que ayude a fortalecer el sentido crítico de los estudiantes ante las distintas fuentes de información a las que accede por medio de la lectura. No obstante, al examinar la realidad de las escuelas, hallamos que la lectura, a pesar de los esfuerzos de docentes y directivos docentes, no juega este papel importante que exige la época actual pues los estudiantes no poseen las habilidades requeridas en cada uno de los niveles de la comprensión de textos.

Por su parte, los docentes, sobre todo en la Educación Básica Primaria, no poseen las herramientas suficientes para acercar al estudiante a los textos y que éste pueda leerlos de manera crítica y comprensiva. El docente de este nivel enfatiza mayormente en el acto de decodificar los mensajes contenidos en los textos y se limita a que se pueda leer de manera fluida (rápida) y se consiga encontrar el tema, siempre y cuando se encuentre de manera literal, con lo cual se deja por fuera del acto lector, la formación del pensamiento, la relación de lo leído con el contexto en el que se escribió y las implicaciones del contenido del texto en la realidad cotidiana. Por esta razón, se hace necesario que el docente tenga una visión diferente del proceso lector, una que le permita proveer a los estudiantes estrategias para enfrentar los textos y llegar a su comprensión.

La presente investigación, buscó realizar un aporte en la formación del profesorado de la Institución Educativa El Viajano, de la zona rural del municipio de Sahagún, teniendo como marco el Programa Todos a Aprender en el cual la investigadora desempeña el papel como tutora. Este programa fue implementado por el Ministerio de Educación para elevar los niveles de la calidad de los procesos educativos en la Educación Básica Primaria, sobre todo en las áreas de Matemáticas y Lenguaje. El trabajo se realiza con los docentes, con los cuales se conforma una Comunidad de Aprendizaje, en la cual se comparten una serie de lineamientos para la preparación de las clases, el fortalecimiento de los procesos de enseñanza y aprendizaje y la socialización de necesidades que puedan ser atendidas desde las bases del programa.

La institución Educativa El Viajano cuenta con 11 sedes, en las cuales se encuentran aulas multigrado, con niños de distintas edades, los docentes de la básica primaria son 30, los cuales tienen formación en distintas disciplinas, normalistas superiores, licenciados en pedagogía reeducativa, preescolar, educación religiosa, básica primaria, entre otros. Hay que anotar que pocos tienen el perfil de lengua castellana, muchos orientan esta asignatura sin un perfil académico, al respecto comenta el docente 1 "se hace lo que se puede y uno va siguiendo la orientación del libro". Por esta razón, la enseñanza de la lectura en este nivel escolar es abordada desde la concepción que cada docente haya construido a lo largo de su formación académica y su experiencia profesional y personal. 
El programa Todos Aprender lleva cinco años en la institución, dentro de las actividades realizadas con los docentes están las Sesiones de Trabajo Situado, Acompañamiento en el Aula y Comunidades de Aprendizaje. Como resultado de las observaciones iniciales, las características de la población y estos encuentros pedagógicos surgió la necesidad de develar y dar cuenta de las concepciones y prácticas de lectura de los docentes de la básica primaria de la institución. Frente a estas dificultades, surge la pregunta que direcciona este estudio de investigación

Además, se plantea como objetivo general el de modelar un sistema de estrategias, desde un enfoque sociocultural, que permita crear una comunidad de maestros lectores en la Institución Educativa El Viajano, partiendo de las comunidades de aprendizaje promovidas por el Programa Todos a Aprender. Con lo cual, se beneficia a los estudiantes, pues sus docentes, convertidos en lectores y transformando sus concepciones sobre los procesos de lectura, llevarán al aula una serie de estrategias de acercamiento a los textos, que posibilitarán la formación de lectores críticos, tal y como lo exige la época actual. Esto último constituye una de las razones por las cuales la realización del estudio se justificó.

\section{Referentes}

Para dar fuerza al proceso de investigación acción, se explora una serie de investigaciones en el ámbito internacional y a nivel nacional, pues es necesario partir del estado del arte del tema que se desarrolla en el trabajo. A continuación, se comparte uno de esos trabajos que se han tomado como antecedentes investigativos.

Entre las investigaciones encontradas en el ámbito internacional se puede destacar la adelantada en el año 2012 por Rosemary Duarte Cunha de la Universidad de Alcalá de España, en la cual se promueve el uso de una metodología específica de la enseñanza de la lectura en las series iniciales de la etapa de aprendizaje de los niños en la escuela, desarrollando así un comportamiento lector desde la infancia. Así mismo, estimula la lectura y planea el desarrollo del comportamiento del lector en la búsqueda de la sostenibilidad social y cultural, promoviendo la reducción de la desigualdad en la sociedad actual, estableciendo la lectura como fuente de conocimiento y de comprensión del mundo. Se utiliza, en este caso, la metodología cualitativa para investigar las prácticas de la lectura y su repercusión en el comportamiento lector.

Este trabajo realizado desde la Universidad de Alcalá de Henares es un estudio cualitativo cuyos objetivos sirven al presente trabajo al incluir el rol del docente como un lector que es el ejemplo a seguir por parte de los estudiantes por cuanto se convierte en un facilitador de estrategias de lectura que tienen como fin la comprensión y análisis de los mensajes contenidos en los textos que se leen. Además, teóricamente concibe que la lectura debe ser enseñada con una metodología específica que promueva el diseño e implementación de estrategias 
surgidas de la reflexión del docente, tal y como se propone desde las comunidades de aprendizaje que se promueven en el Programa todos a Aprender.

Al estar basado en la interacción de los sujetos participantes en los procesos de aula, el programa permitió que se trabajara desde un enfoque socio cultural la enseñanza de las estrategias de lectura, pues como ya se ha señalado, desde esta perspectiva se ve a la lectura como una práctica social que permite a los sujetos participantes en las situaciones comunicativas, en este caso, la comunidad lectora y el aula de clases, mejorar la comprensión lectora desde la comprensión de la realidad misma. En palabras de Freire (1989): "Leer no consiste solamente en decodificar la palabra o el lenguaje escrito; antes bien es un acto precedido por (y entrelazado) el conocimiento de la realidad" (P.51).

Teniendo en cuenta estos aspectos, las estrategias planteadas por el Programa Todos a Aprender (PTA), fueron pensadas desde este enfoque sociocultural, por lo cual, para el presente estudio, se enfatizó en aquellas que posibilitaron una relación del docente con sus estudiantes, generando en ellos la formación de sujetos autónomos que puedan establecerse metas y propósitos para afrontar las relaciones con otros agentes sociales, en este caso, sus propios compañeros y la realidad que los circunda. Por lo tanto, el sustento teórico de la presente investigación parte de la concepción de que la lectura debe ser enseñada por medio de metodologías que permitan el diseño de estrategias que puedan ser enseñadas a los estudiantes pues como lo afirma James P. Gee: “Cada tipo de texto puede leerse de varios modos; el significado puede tomarse del texto o aportarse al texto en varios niveles. [...] Los tipos de texto y las sus distintas maneras de leerlos no fluyen en un soplo del alma individual (o de su biología); son la invención social e histórica de varios grupos sociales".

Esto es fundamental para entender el asunto, desde la interacción social deben plantearse las soluciones pues ya no es un asunto de resolver pruebas de lectura, sino que es la exigencia del mundo actual sobre los individuos, la lectura comprensiva es una competencia muy necesaria en las actuales circunstancias.

La visión sociocultural sustenta que leer y escribir son actividades culturales situadas en un contexto social que varían de acuerdo con cada época y el lugar o comunidad donde se desarrollan; de esta manera cada comunidad desarrollará sus propias prácticas letradas, así como las disciplinas científicas tienen a su vez rasgos propios que las distinguen. La comunidad educativa de la Institución El Viajano es un caso particular, por lo tanto, un paso importante en este trabajo es observar la forma en que las prácticas sociales influyen sobre los niños y cómo llevan al aula las necesidades que tiene para poder interactuar en sus diferentes entornos.

Una perspectiva sobre la lectura que se ha intensificado en los últimos años es el enfoque sociocultural, desde la perspectiva del trabajo por proyectos, por esta cuestión queremos tomar nuestro punto de partida desde los trabajos de Casssany (2006), cuando dice que nos debemos enfrentar a un texto como personas, no en forma mecánica, con tareas que permitan utilizar mecanismos y procedimientos con los que leemos en el mundo real. Este autor pone el énfasis en el enfoque sociocultural, nos habla sobre las prácticas de lectura y plantea que leer y escribir no son sólo tareas lingüísticas o procesos psicológicos, sino también 
prácticas sociales puesto que asumimos unos roles predeterminados de "lector" y "autor": Ios alumnos llegan a la escuela con una serie de conocimientos previos y con información variada.

Es indudable que lo que señalan Cassany y Castellá (2011) con respecto a la evolución de las prácticas lectoras se comprueba día a día en nuestra vida cotidiana; nadie es un letrado absoluto en el sentido de haber leído todos los textos escritos y el conocimiento del idioma no garantiza que podemos utilizar todas las prácticas lectoras; así mismo la cantidad de textos que se publican es enorme y mayor aún la cantidad de los que se pueden disponer en Internet. Pero es necesario añadir nuevamente que el entorno influye y que sólo podemos dominar cierta cantidad de textos por lo que es indudable que debemos ser capaces de seleccionar los textos, leer críticamente, identificar la ideología y descubrir lo que se oculta tras las líneas.

Por otra parte, este trabajo se configuró a partir de Comunidades de Aprendizaje (CDA), entendidas como "un proyecto de transformación social y cultural de un centro educativo y de su entorno, para conseguir una sociedad de la información para todas las personas" (Valls, 2005). Por eso, para comprender este trabajo, es necesario conocer la estrategia de trabajo de los docentes que conforman una CDA en las instituciones educativas oficiales, con el propósito de identificar las prácticas pedagógicas, planear acciones encaminadas hacia la mejora de los procesos académicos de una institución y de esta manera, promover dinámicas de trabajo para fortalecimiento de los aprendizajes de los estudiantes (Vargas, 2018).

Algunos autores destacan que las CDA de docentes son efectivas en el desarrollo profesional del docente y liderazgo escolar (Au, Hu-pei, 2002; CochranSmith y Lytle, 1999; Fullan, 2005.) La CDA de docentes provee un ambiente fértil para desarrollar actividades de aprendizaje colaborativo entre docentes, que conllevan a desarrollar procesos de investigación y acción, como también procesos de autoevaluación. Gran parte de los resultados conducen a mejoras en las prácticas de enseñanza y de evaluación formativa en el aula que pueden mejorar el rendimiento académico estudiantil (Vargas, 2018; Roberts \& Pruitt, 2008; DuFour \& Eaker, 1998; Calhoun, 1994).

De acuerdo con esto, la CDA es un espacio de interacción que permite la aplicación del enfoque sociocultural pues dicha comunidad de aprendizaje se ha convertido en una realidad social que enfrentan los docentes que participaron en el estudio. La CDA es entonces, un espacio de interacción entre pares, en el cual se discuten asuntos relacionados con la enseñanza de la lengua, lo que permite la transformación de ésta en una comunidad de docentes lectores. Esto significa que la comunidad lectora se basa en la experiencia de comunicación social entre los participantes, donde tienen la oportunidad de acceder a nuevos conocimientos, a las experiencias de los demás y de esta manera llegar a autocuestionarse sobre su desempeño.

En este contexto, una comunidad de docentes lectores es un escenario en el que sus participantes comparten lecturas de diferentes tipos de textos, se promueve la reflexión de los contenidos y mensajes esenciales del texto y luego se presentan diferentes apreciaciones acerca de las posibilidades de llevar estos 
ejercicios y estrategias de lectura al aula, con el fin de favorecer el desarrollo de habilidades de lectura comprensiva en los estudiantes de una institución educativa.

\section{Metodología}

Para enfrentar un estudio que permita el desarrollo de una comunidad de docentes lectores que puedan llevar estrategias de lectura a sus respectivas aulas en la Institución Educativa El Viajano, se orientó este trabajo desde el paradigma cualitativo, con el método de investigación acción, el cual facilita la comprensión de lo que ocurre en el contexto estudiado y sus resultados permiten diseñar e implementar las acciones necesarias para generar los cambios y mejoras que el problema en estudio amerita. Los participantes, fueron 6 docentes seleccionados de manera aleatoria de un total de 30, que estaban focalizados por el programa Todos a Aprender en la institución educativa.

El estudio se desarrolló teniendo en cuenta cuatro fases propuestas por Carr y Kemis (1988): planificación, en el cual, partiendo de la observación, se realizó una caracterización de la población y se formuló la ruta a seguir y los instrumentos empleados en cada una de ellas; acción, en el cual se realizaron los grupos de discusión y entrevistas no estructuradas y se compartieron las estrategias de abordaje de la lectura; observación, es la fase donde se registraron en el diario de campo los desempeño y las voces de los docentes durante las jornadas de formación en el marco de las comunidades de aprendizaje y la puesta en escena de las estrategias de aula compartidas; y, por último, la fase de reflexión, en el cual se analizó toda la información y se elaboró el informe de investigación.

Estas fases permitieron partir de la observación y análisis de la realidad vivida en el aula con respecto a la enseñanza y uso de la lectura por parte de los docentes participantes, pasando por la formulación y adaptación de estrategias de lectura compartidas en la comunidad de aprendizaje que se llevan a cabo en el marco del Programa Todos a Aprender (PTA) y llegando finalmente a observar en las clases la implementación de dichas estrategias, lo cual dio como resultado un análisis de la información levantada que da cuenta del impacto que generó la comunidad de docentes lectores en el acto lector de sus estudiantes.

A nivel general, esta investigación representa un aporte a la comprensión de que la existencia de factores diversos dentro de los salones de clase tales como las variadas formas de lenguaje, las actitudes, las expectativas, la formación profesional de los maestros, las historias académicas, las experiencias pedagógicas, las metodologías de enseñanza, los contenidos temáticos, los diagnósticos y planes institucionales dan cuenta de la imposibilidad de contar con un método único y exclusivo de aproximación al conocimiento en el aula. Por lo que fue necesario el uso de diferentes enfoques, metodologías, técnicas e instrumentos que permitieran producir saberes en el complejo mundo social del aula. 


\section{Técnicas e instrumentos de recolección de información}

En esta investigación se utilizaron diferentes técnicas e instrumentos de recolección y análisis de información. Básicamente los instrumentos empleados para el registro de los datos y observaciones, fueron adaptados a partir de los que utilizados en el programa Todos a Aprender. Específicamente, se utilizaron los siguientes instrumentos:

La observación participante: esta técnica permite la interacción social del observador en el escenario con los sujetos observados, además faculta a los investigadores a aprender acerca de las actividades de las personas en estudio en un escenario natural a través de la observación y participando en sus actividades (DeWalt \& DeWalt, 2002), es decir, se trata de hacer la observación participando en todas las actividades como miembro del grupo, la gran ventaja de ejercer este rol es que permite que se pueda hacer parte activa del contexto al involucrarse en las actividades de rutina de los participantes en el escenario del investigador (Schensul, Schensul, \& LeCompte, 1999). El uso de esta técnica, permitió obtener la mayor cercanía posible con los participantes, para describir las situaciones observadas y tener un mayor conocimiento que posteriormente permita la transformación de la realidad.

Grupo de discusión: esta técnica metodológica se ocupa del estudio de lo que piensan, lo que comparten varios individuos en un proceso de reflexión construida en un espacio común. De este modo, con el grupo de discusión los participantes pudieron reflexionar al inicio de la investigación con respecto a sus concepciones y la importancia de la lectura en el aula, y más adelante, se reflexiona en torno a la pertinencia e impacto de las estrategias de lectura aplicadas en la comunidad de docentes lectores (CDL).

Entrevista no estructurada: desde el enfoque de la investigación-acción, la entrevista no estructurada ofrece la ventaja de ser una conversación en la que se busca la comprensión del otro frente al tema o fenómeno estudiado. Taylor y Bogdan (1987) entienden la entrevista en profundidad como reiterados encuentros cara a cara entre el entrevistador y los informantes, dirigidos hacia la comprensión de las perspectivas que tienen los informantes respecto de sus vidas, experiencias o situaciones, tal y como las expresan con sus propias palabras. En esta investigación, se empleó esta técnica en la fase de observación para reconocer algunos elementos de la problemática en cuestión, pues se requería que los docentes focalizados hablaras desde su experiencia, sobre la enseñanza de la lectura. Además, en la fase de acción, también se realizaron entrevistas para establecer las reflexiones de los participantes en la comunidad lectora con respecto a las estrategias empleadas

\section{Categorías de Análisis}

En este estudio se desarrolló un trabajo de campo que permitiera analizar la información que se recopilaba en cada fase, de tal modo que se pudiera obtener una mayor comprensión e interpretación de las situaciones que ocurren en el contexto real. A partir de esto, se obtuvieron las siguientes categorías: 
Tabla 1: Categorías de Análisis.

\begin{tabular}{|c|c|c|c|c|}
\hline Preguntas & Objetivos específicos & Categorías & Rasgos & Descriptores \\
\hline $\begin{array}{l}\text { ¿Qué concepciones y } \\
\text { prácticas de lectura } \\
\text { aplican los docentes de } \\
\text { Educación Básica } \\
\text { Primaria de la } \\
\text { Institución Educativa El } \\
\text { Viajano del Municipio } \\
\text { de Sahagún? }\end{array}$ & $\begin{array}{lr}\text { Reconocer } & \text { las } \\
\text { concepciones } & \text { y } \\
\text { prácticas de lectura } \\
\text { que aplican los } \\
\text { docentes de Básica } \\
\text { Primaria de la } \\
\text { Institución Educativa El } \\
\text { Viajano del Municipio } \\
\text { de Sahagún. }\end{array}$ & $\begin{array}{l}\text { Concepcione } \\
\text { s y prácticas } \\
\text { de lectura }\end{array}$ & $\begin{array}{l}\text { Conceptos } \\
\text { Importancia } \\
\text { de la lectura y } \\
\text { su enseñanza }\end{array}$ & $\begin{array}{l}\text { Conceptos sobre } \\
\text { la lectura } \\
\text { Juicios sobre la } \\
\text { importancia de } \\
\text { lectura } \\
\begin{array}{l}\text { Prácticas de } \\
\text { enseñanza de la } \\
\text { lectura. }\end{array}\end{array}$ \\
\hline 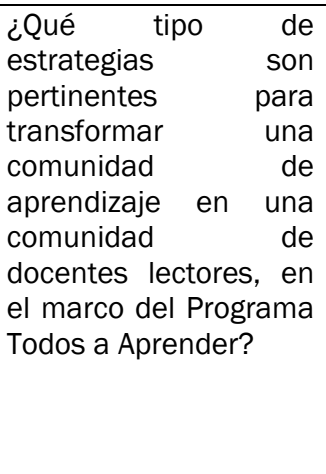 & $\begin{array}{lr}\text { Diseñar una tipología } \\
\text { de } & \text { estrategias } \\
\text { didácticas, r con } \\
\text { enfoque socio-cultural, } \\
\text { que al ser aplicadas } \\
\text { orienten r la } \\
\text { transformación de una } \\
\text { comunidad r de } \\
\text { aprendizaje en una } \\
\text { comunidad } \\
\text { docentes lectores, en } \\
\text { el marco del Programa } \\
\text { Todos a Aprender. }\end{array}$ & $\begin{array}{l}\text { Estrategias } \\
\text { didácticas } \\
\text { con enfoque } \\
\text { sociocultural }\end{array}$ & $\begin{array}{l}\text { Acciones } \\
\text { para } \\
\text { promover la } \\
\text { lectura en la } \\
\text { escuela y la } \\
\text { vida personal }\end{array}$ & $\begin{array}{l}\text { Estrategias en la } \\
\text { comunidad de } \\
\text { docentes lectores } \\
\text { (CDL) } \\
\text { Estrategias } \\
\text { empleadas en el } \\
\text { aula }\end{array}$ \\
\hline $\begin{array}{l}\text { ¿Qué cambios se } \\
\text { generan en las } \\
\text { prácticas de lectura de } \\
\text { los docentes de la } \\
\text { Institución Educativa el } \\
\text { Viajano al participar en } \\
\text { la comunidad de } \\
\text { docentes lectores } \\
\text { (CDL)? }\end{array}$ & $\begin{array}{l}\text { Analizar los cambios } \\
\text { que se generan en los } \\
\text { docentes y r sus } \\
\text { prácticas de aula a } \\
\text { partir de } \quad \text { la } \\
\text { participación en la } \\
\text { comunidad rra } \\
\text { docentes lectores } \\
\text { (CDL). }\end{array}$ & $\begin{array}{l}\text { Transformaci } \\
\text { ones en la } \\
\text { lectura y la } \\
\text { enseñanza de } \\
\text { la misma }\end{array}$ & $\begin{array}{l}\text { Acciones } \\
\text { realizadas } \\
\text { por los } \\
\text { docentes en } \\
\text { su } \\
\text { desempeño } \\
\text { personal y } \\
\text { profesional }\end{array}$ & $\begin{array}{l}\text { Participación en } \\
\text { la comunidad de } \\
\text { docentes lectores } \\
\text { (CDL) } \\
\text { Implementación } \\
\text { de estrategias en } \\
\text { la práctica } \\
\text { docente. }\end{array}$ \\
\hline
\end{tabular}

Fuente: Balmaceda (2018).

\section{Resultados}

Los resultados de cada una de las fases de la investigación se presentan en tres unidades de análisis, organizadas desde cada uno de los objetivos y las categorías asociadas a estos: concepciones de docentes sobre la lectura, su importancia y enseñanza, estrategias didácticas de lectura y transformaciones en la práctica docente. 
En la primera categoría, relacionada con las concepciones e importancia de que le dan los docentes a la lectura y sus prácticas, encontró que los docentes reconocen la importancia de la lectura, al llevar al aula actividades relacionadas con ella, sin embargo, no se evidencia mayor esfuerzo para potenciar habilidades en los estudiantes, hay poca interacción entre el docente, el texto y el niño lector, los docentes emplean los procesos de lectura en un contexto mayormente evaluativo. Con respecto a las acciones llevadas a cabo para promover la lectura, los docentes, en general, dan el tradicional discurso de que los estudiantes son los responsables de los bajos resultados, frente a lo cual nunca se plantea la necesidad de revisar las prácticas y concepciones de los docentes frente a los procesos de enseñanza y aprendizaje de la lectura.

De igual forma en el grupo de discusión, se evidencia de diferente manera una misma realidad; se concibe la lectura como una herramienta cognitiva y cognoscitiva que les permite a los estudiantes un mayor acceso al conocimiento del mundo, a su formación integral y que por lo tanto es importante. Esto se sustenta en algunas afirmaciones que hacen los docentes, por ejemplo: “... la lectura hace parte de la formación integral de los niños, al comprenderla e interpretarla un niño da mejores resultados". Aquí se demuestra que los docentes usan la lectura mayormente en el contexto evaluativo y no formativo.

A nivel general, las concepciones de los docentes con respecto a la lectura y su importancia, evidencian un nivel alto de reconocimiento de ésta como un vehículo que permite a los niños transformarse y ser agentes de cambio en la sociedad, un participante afirma que "La lectura sin duda cumple el papel más importante en la formación de los niños, si no se incentiva el amor hacia ella no podríamos hablar de niños lectores". Esta es una conclusión que muchos de ellos confirman, la formación de lectores depende de los docentes y sus prácticas de lectura.

En la segunda categoría, se hace referencia a las acciones que se realizan para direccionar los procesos de lectura en el aula y en la vida personal, por lo que la información se analizó desde dos puntos de vista, por un lado, las estrategias empleadas en el aula por lo docentes, y, por otro, las que se aplicaron al interior de la CDA para convertir este espacio en una CDL. De acuerdo con las respuestas dadas por los participantes, existe un reconocimiento de la importancia de la lectura en el proceso académico por lo que la incluyen en diferentes momentos de la jornada escolar. No obstante, no se evidencia una estructuración del trabajo que permita a los estudiantes ir desarrollando las habilidades que se requieren en cada tipo de texto. Por otro lado, los docentes buscan que se lea y se respondan preguntas, haciendo de la clase de lectura un proceso mecanizado en el que los estudiantes participan, pero no se atienden sus necesidades específicas en cuanto a formación como lectores ya que las estrategias hacen mayor énfasis en la evaluación que en la formación.

Partiendo de este análisis, además de los que se han realizado en cuanto a las prácticas de lectura de los docentes, se identificaron del tipo tradicional y constructivistas, en las cuales se busca como principal propósito un énfasis en la apropiación del texto a nivel estructural y con el fin de hallar el mensaje contenido en ideas principales y secundarias, tipos de narrador y los personajes. Por todos 
estos aspectos, se hizo necesaria la formación de una comunidad de docentes lectores (CDL) para que desarrollara estrategias pedagógicas con un enfoque sociocultural de la lectura, y se posibilitaran cambios en el desempeño lector de los docentes y en las prácticas de enseñanza que venían realizando.

Con la realización de estrategias al interior de la CDA, se consiguió que los docentes participaran activamente, reconociendo la importancia de ellas, inicialmente para su propio proceso lector. Aunque es importante señalar que al inicio se notó algo de resistencia, los docentes se fueron motivando a participar en las lecturas en voz alta, algunos coordinando las estrategias, trayendo o sugiriendo materiales de lectura y realizando comentarios pertinentes sobre la aplicabilidad de estas estrategias en el aula para conseguir la mejora en el proceso lector de los estudiantes a partir de la interacción entre ellos y con el docente alrededor de ejercicio de lectura que permitan la interacción no solo con el texto, sino con el contexto y las apreciaciones y reflexiones de los demás lectores.

Por último, con relación a la tercera categoría se detallan las transformaciones observadas alrededor de dos aspectos. En primer lugar, con respecto a la participación en las comunidades de docentes lectores, y, además, el uso de las estrategias en el desempeño del docente en el aula. En cuanto al análisis de la participación de los docentes en las estrategias que se diseñaron e implementaron al interior de la CDL, es importante resaltar el hecho de que al principio un número considerable de ellos no participó activamente en las lecturas $\mathrm{ni}$ en las discusiones, sin embargo, luego se mostraron muy receptivos y participativos al asumir el rol de estudiantes y establecieron lazos de trabajo colaborativo, lo que permitió un clima de confianza y respeto frente a los demás y frente a las metas establecidas en el grupo de trabajo.

Un participante expresa "se podrá mejorar la lectura con los estudiantes si se promueve el trabajo en equipo y los juegos a partir de lecturas escogidas", otro docente manifiesta "lo que más me gusta es que ahora selecciono estudiantes para leer individual y luego ellos comparten conmigo la experiencia mientras los demás escuchan y se motivan", mientras que una docente resalta la necesidad de "documentar estas actividades para llevarlas a la lectura en el aula". Estas afirmaciones realizadas en los procesos de reflexión al finalizar la aplicación de las estrategias, dan cuenta no sólo de la participación activa de los docentes en la comunidad, sino de las transformaciones que se presentan en la concepción de la lectura que se tenía anteriormente, pues ya no se piensa en que el docente de lenguaje sea quien promueva la lectura, sino que se asume el compromiso de todos para consolidarse como un docentes lectores y llevar a cabo esta tarea en las aulas de clase.

El trabajo desarrollado, demostró que los docentes participantes implementan diferentes formas de abordar la lectura en la planeación de clases, aplican estrategias de las que se compartieron en el grupo de trabajo, las combinan de diversas maneras, o crean nuevas formas ideadas por ellos para asumir de forma diferente la lectura en el aula. 


\section{Assensus}

Revista de Investigación educativa y pedagógica

\section{Conclusiones}

Los resultados de este estudio demuestran que la investigación acción se consolida como un método de gran valor en el proceso de formación y actualización docente, puesto que permite la reflexión crítica constante de su labor pedagógica en el aula. Además, la conformación de un equipo de trabajo, la distribución de responsabilidades y sobre todo, la reflexión colectiva sobre su práctica, se convirtieron en elementos esenciales en la búsqueda de la calidad del proceso de aprendizaje.

En este sentido, la transformación de la comunidad de aprendizaje en una comunidad de docentes lectores fue posible con las estrategias implementadas en esta investigación, las cuales se enmarcan en el enfoque sociocultural, desde el cual se posibilitan espacios de interacción entre los sujetos participantes que comparten sus experiencias de lectura y en ese diálogo de saberes y formas de leer, potenciando en cada participante sus habilidades y capacidades para la enseñanza y didáctica de la lectura, lo cual sin duda impacta su desempeño en favor de sus estudiantes.

El análisis de la información derivada de esta investigación se pudo evidenciar que los docentes juegan un papel fundamental en la formación de niños lectores, pues es el docente el que irradia en el aula ese espíritu lector, esa ganas de encontrar en la lectura mundos inimaginables, es el guía y conductor de un sentido humanista encontrado en las letras y fundamentado en el conocimiento de su disciplina, esto, unido a unas buenas prácticas de lectura por parte del docente, hará de su quehacer pedagógico una mejor práctica docente.

Por otra parte, la creación de la comunidad lectora en el marco de la CDA del Programa Todos Aprender sirvió de escenario común para llegar a acuerdos y reflexiones de tipo pedagógico que sirvieron de base para el análisis de las prácticas de lectura empleadas por los docentes de la institución. En esas interacciones se evidenció un interés por conocer y apropiarse de los fundamentos teóricos y didácticos en los que se fundamenta la lectura, con el fin de actualizar sus programas académicos, proyectando su aprendizaje a los estudiantes en el aula escolar. Se demuestra entonces, que el Programa Todos a Aprender es una herramienta valiosa en la consecución de resultados institucionales pues la intervención de los procesos de aula es benéfica para las comunidades educativas cuando se articulan los objetivos de la enseñanza. 


\section{Referencias}

Au, Kathryn Hu-pei. (2002) Communities of Practice: Engagement, Imagination, and Alignment in Research on Teacher Education. Journal of Teacher Education 53 (3).

Calhoun, E. (1994) How to use action research in the self-renewing school. Alexandria, VA: Association for Supervision and Curriculum Development.

Cassany, D. (2006) Tras las líneas. Sobre la lectura contemporánea. 294 pp. Barcelona: Editorial Anagrama S.A. ISBN: 84-339-6236-1

Cassany D, Castellá J. (2011). Aproximación a la literacidad crítica. Perspectiva. 28(2), pp. 353374.

Cochran-Smith, Marilyn, \& Lytle, Susan S. (1999) "Relationship of Knowledge and Practice: Teacher Learning in Communities." In Review of Research in Education, Vol. 24, ed. Ali Iran-Nejad and P. David Pearson. Washington, DC: American Educational Research Association.

DeWalt, K., \& DeWalt, B. (2002). Participant observation: a guide for fieldworkers. Walnut Creek, CA: AltaMira Press.

DuFour, R. \& Eaker, R. (1998) Professional learning communities at work: Best practices for enhancing student achievement. Bloomington, IN: National Educational Service and Alexandria, VA: Association of Supervision and Curriculum Development.

Freire, P. (1989). La educación como práctica de la libertad. Madrid: Siglo XXI.

Fullan, M. (2005) Leadership and sustainability: system thinkers in action. Thousand Oaks, CA: Corwin Press \& Toronto, ONT: Ontario Principals' Council.

Roberts, S. M. \& Pruitt, E. Z. (2003). Schools as professional learning communities: Collaborative activities and strategies for professional development. Thousand Oaks, CA: Corwin

Schensul, S., Schensul, J., \& LeCompte, M. (1999). Essential ethnographic methods: Observations, interviews, and questionnaires (Book 2 en Ethnographer's Toolkit). Walnut Creek, CA: AltaMira Press.

Taylor, S., \& Bogdan, R. (1987). Introducción a los métodos cualitativos de investigación. Barcelona: Paidós.

Valls, R. (2005). Los educadores y las educadoras sociales en las comunidades de aprendizaje. Revista de educación social, (4). Recuperado el 13 de mayo de 2018, de http://www.eduso.net/res/?b=7\&c=53\&n=145

Vargas, J. (2018). Las comunidades de aprendizaje y la evaluación formativa: Una experiencia significativa para transformar la práctica docente y el aprendizaje de los estudiantes desde el programa Todos a Aprender. Assensus, 3(5), 44-59. Recuperado de https://revistas.unicordoba.edu.co/index.php/assensus/article/view/1512 